\title{
The nature of auditory discrimination problems in children with specific language impairment: An MMN study
}

\author{
Nina Davids ${ }^{\mathrm{a}, \mathrm{b}, *}$, Eliane Segers ${ }^{\mathrm{a}}$, Daniëlle van den Brink ${ }^{\mathrm{b}, \mathrm{c}}$, Holger Mitterer $^{\mathrm{d}}$, Hans van Balkom $^{\mathrm{a}}$, \\ Peter Hagoort ${ }^{\mathrm{b}, \mathrm{d}}$, Ludo Verhoeven ${ }^{\mathrm{a}}$ \\ a Behavioural Science Institute, Radboud University Nijmegen, The Netherlands \\ ${ }^{\mathrm{b}}$ Donders Institute for Brain, Cognition and Behaviour, Radboud University Nijmegen, The Netherlands \\ c Radboud University Nijmegen Medical Centre, The Netherlands \\ ${ }^{\mathrm{d}}$ Max Planck Institute for Psycholinguistics, The Netherlands
}

\section{A R T I C L E I N F O}

\section{Article history:}

Received 3 February 2010

Received in revised form 1 November 2010

Accepted 3 November 2010

Available online 9 November 2010

\section{Keywords:}

Mismatch negativity (MMN)

Event-related potential (ERP)

Specific language impairment (SLI)

Phonemic discrimination

\begin{abstract}
A B S T R A C T
Many children with specific language impairment (SLI) show impairments in discriminating auditorily presented stimuli. The present study investigates whether these discrimination problems are speech specific or of a general auditory nature. This was studied using a linguistic and nonlinguistic contrast that were matched for acoustic complexity in an active behavioral task and a passive ERP paradigm, known to elicit the mismatch negativity (MMN). In addition, attention skills and a variety of language skills were measured. Participants were 25 five-year-old Dutch children with SLI having receptive as well as productive language problems and 25 control children with typical speech- and language development. At the behavioral level, the SLI group was impaired in discriminating the linguistic contrast as compared to the control group, while both groups were unable to distinguish the non-linguistic contrast. Moreover, the SLI group tended to have impaired attention skills which correlated with performance on most of the language tests. At the neural level, the SLI group, in contrast to the control group, did not show an MMN in response to either the linguistic or nonlinguistic contrast. The MMN data are consistent with an account that relates the symptoms in children with SLI to non-speech processing difficulties.
\end{abstract}

(c) 2010 Elsevier Ltd. All rights reserved.

\section{Introduction}

Children with specific language impairment (SLI) fail to develop normal language, which cannot be explained by a general cognitive impairment, hearing problems, major neurological abnormalities, and/or abnormal behavior (Bishop, 1992; Leonard, 1998). They often have problems in speech comprehension, such as phonological processing of auditory information (Bishop, 1997; Leonard, 1998). For instance, children with SLI have difficulties in discriminating stimuli that are minimal pairs (e.g., /ba/vs./da/). These discrimination problems are related to a phonological deficit; an impairment in representing, storing, and/or retrieving phonemes (Snowling, 2000). A phonological deficit interferes with the ability to learn the correspondences between phonemes and graphemes and hence many children with SLI develop difficulties in learning to read (Bird, Bishop, \& Freeman, 1995; Bishop \& Adams, 1990). To better understand the nature of these auditory dis-

\footnotetext{
* Corresponding author at: Behavioural Science Institute, Radboud University Nijmegen, P.O. Box 9104, 6500 HE Nijmegen, The Netherlands. Tel.: +31 243612822; fax: +31243616211.

E-mail address: nina.davids@berkeley.edu (N. Davids).
}

crimination problems, many studies investigated whether these problems are primarily speech specific, or whether they coincide with non-speech processing difficulties. This has been studied both in children with SLI and in children with dyslexia, since phonological problems are present in both groups (Bishop \& Snowling, 2004). However, due to inconclusive results questions regarding the specific nature of auditory discrimination problems remain unanswered (Ramus, 2001; Ramus et al., 2003; Rosen, 2003). The present study was designed to shed more light on the nature of these auditory discrimination problems, by using equally complex speech and non-speech contrasts in a behavioral study and a mismatch negativity (MMN) study in which the brain's automatic responses are measured.

In the past, several behavioral studies have obtained evidence for a primarily phonological and speech specific deficit in children with SLI and/or dyslexia, supporting theories such as the phonological deficit theory (e.g., Mody, Studdert-Kennedy, \& Brady, 1997; Snowling, 2000; Studdert-Kennedy, 2002; StuddertKennedy \& Mody, 1995). For example, Mody et al. (1997) showed that reading impaired children had more difficulties than controls discriminating speech sounds but this was not the case with non-speech sounds. However, other studies have suggested that the phonological deficit is caused by a more general prob- 
lem in processing non-speech sounds (e.g., Farmer \& Klein, 1995; McAnally, Castles, \& Stuart, 2000; Stein \& Walsh, 1997; Tallal \& Piercy, 1973a, 1973b, 1974, 1975). Tallal and Piercy (1973a, 1973b, 1974, 1975), for example, showed that children with SLI, referred to by them as children with developmental aphasia and developmental dysphasia, display problems with both speech and non-speech stimuli containing short and/or rapid acoustic changes.

A limitation of these behavioral studies is that the results may have been influenced by attention problems of the participants. To accomplish a behavioral task, attention and motivation is required, and around $35 \%$ of all children with speech and language disorders have been shown to suffer from an attention disorder (Beitchman, Hood, \& Inglis, 1990). Other studies have investigated the nature of auditory discrimination problems by means of a passive oddball paradigm measuring event-related brain potentials (ERPs). In such a paradigm no attention to the auditory stimuli, no instruction, and no motor or verbal response is required. In these studies, the ERP of interest was the MMN, which has been shown to be indicative of auditory and phonemic discrimination processing at a preconscious level (Näätänen, Gaillard, \& Mantysalo, 1978; Näätänen, Paavilainen, Rinne, \& Alho, 2007).

Most MMN studies that have investigated the nature of a phonological deficit in children with SLI and/or dyslexia suggest phonological deficits do not coincide with non-speech processing difficulties (Meng et al., 2005; Schulte-Körne, Deimel, Bartling, \& Remschmidt, 1998; Schulte-Körne, Deimel, Bartling, \& Remschmidt, 1999; Sharma et al., 2006; Uwer, Albrecht, \& von-Suchodoletz, 2002). For example, Uwer et al. (2002) showed that eight-year-old children with SLI showed smaller MMNs for linguistic contrasts (/da/vs./ga/and/ba/) than control children without any language problems. No group differences were found for non-linguistic contrasts in tone frequency and duration $(1000 \mathrm{~Hz}$ vs. $1200 \mathrm{~Hz}$; $175 \mathrm{~ms}$ vs. $100 \mathrm{~ms}$, respectively). Nor were differences found between the MMNs of children with receptive SLI and children with expressive SLI. It should be mentioned that, in their study, rather late and long MMN latency windows were used for the statistical analysis. In contrast, recent findings from Lachmann, Berti, Kujala, and Schröger (2005) provided evidence for general non-speech processing difficulties. They found that, in contrast to control children, 8- to 11-year-old dyslexic children having difficulties with reading mainly high-frequent words did not show MMNs in a typical MMN latency window for both speech and non-speech contrasts (/ba/vs./da/; $700 \mathrm{~Hz}$ vs. $770 \mathrm{~Hz}$, respectively).

However, in the MMN studies described above the nonlinguistic contrasts were acoustically far less complex than the linguistic contrasts. Whereas linguistic contrasts consisted of rapid changes in several formant frequencies, non-linguistic contrasts consisted of stimuli with stable frequency contours. Therefore, a direct comparison between the phonemic and auditory discrimination abilities using these contrasts may be confounded by acoustic complexity differences (cf. Bishop, 2007; Serniclaes, SprengerCharolles, Carre, \& Demonet, 2001). Alonso-Búa, Díaz, and Ferraces (2006) used more complex non-linguistic stimuli. Speech stimuli in their study consisted of a / ba/-/da/ contrast and the non-linguistic stimuli of short complex tones consisting of five concatenated simple tones. Their results revealed no group differences in MMN amplitude in the typical MMN latency window between 7- to 11-year-old children with reading problems and controls in both linguistic and non-linguistic conditions. In a later time window from 350 to $550 \mathrm{~ms}$, the children with reading problems did show smaller negativities for both linguistic and non-linguistic conditions than controls. Whereas this non-linguistic contrast was more complex than the non-linguistic contrasts used in previous studies, it was still less complex than the linguistic contrast. In a behav- ioral study, Serniclaes et al. (2001) used identical contrasts for the speech and non-speech condition, where the formant frequencies of /ba/and/da/ were replaced by pure tones. In the non-speech condition, participants were told the stimuli were electronic whistles, and in the speech condition they were told that the stimuli were speech-like sounds. Their results revealed that, while fiveyear-old children with dyslexia were less categorical in the speech condition, they were better at discriminating within-categorical contrasts than average readers. Similar results were found in the non-speech condition. This provides evidence that children with a phonological deficit process linguistic as well as non-linguistic information differently as compared to children without phonological problems. Unfortunately, discrimination abilities were not assessed in a passive paradigm, making it impossible to elucidate the role of individual's attention skills in the behavioral results.

Bishop (2007) provided an in-depth review about the electrophysiological assessment of perception abilities in dyslexic and language impaired children. Building upon a suggestion emerging out of this review, here we investigated whether auditory discrimination problems in children diagnosed with SLI are primarily phonological, or whether they coincide with non-speech processing difficulties using speech and non-speech contrasts that have similar acoustically complex changes, in both an active behavioral task and a passive MMN paradigm. This was examined in five-year-old Dutch children diagnosed with SLI. We administered several language tests to assess their phonological abilities. In addition, an attention task was administered to study whether behavioral measures were influenced by attention skills. In the active discrimination experiment (Experiment 1), the nature of discrimination problems was investigated by means of a task in which children had to point to pictures to indicate which of the two stimuli they had heard. In the MMN experiment (Experiment 2 ), linguistic and non-linguistic discrimination abilities were tested passively, using a classic oddball paradigm. In this paradigm, no attention to the auditory stimuli was required, and participants were shown a silent movie. The results of the children with SLI were compared to children with typical speech- and language development.

The main experimental question in the present study is whether discrimination problems of children with SLI are primarily phonological in nature or whether they coincide with non-speech processing difficulties. For the active behavioral task, two different outcomes are possible. First, if discrimination problems are purely phonological, the SLI group should perform below the control group on the linguistic discrimination task, whereas the SLI group should show similar results for the non-linguistic discrimination task when compared to the control group. Second, if children with SLI suffer from non-speech processing difficulties that may affect linguistic information processing, the SLI group should score lower than the control group on both linguistic and non-linguistic discrimination tasks. However, if results from the attention task show that attention skills play a significant role when discrimination abilities are tested behaviorally, then measuring discrimination skills at a pre-attentive level using an MMN paradigm would be necessary to fully characterize discrimination problems in this clinical group. There are two potential outcomes of this study. First, if discrimination problems are restricted to phonological information, the SLI group should show an absence of or a smaller MMN in the linguistic condition as compared to the control group, whereas an MMN comparable to the control group should be observed in the non-linguistic condition. Second, if the discrimination problems of the SLI group are not restricted to phonological information but have a more general nature, the SLI group should show an absence of or smaller MMNs in both conditions as compared to the control group. 


\section{Experiment 1}

\subsection{Method}

\subsubsection{Participants}

Twenty-five children diagnosed with $\mathrm{SLI}^{1}$ ( 9 female, 16 male) with a mean age of 5 years and 9 months $(S D=0.5$, range 4 years and 11 months to 6 years and 5 months) participated in the experiment. This ratio of male to females is common in SLI (Robinson, 1991). Children with SLI were selected if they had both receptive and productive language problems but had not been diagnosed with an autism spectrum disorder. Twenty children with SLI were right-handed and five were left-handed, based on an adapted version of the questionnaire by Oldfield (1971). The children with SLI attended kindergarten at special SLI schools, where SLI had been diagnosed by an interdisciplinary team consisting of clinical linguists and school psychologists. The diagnosis of SLI had been given to children who exhibited a significant deficit in the production and/or comprehension of language that could not be ascribed to general cognitive impairment, sensorimotor deficits, neurological disorders, psychiatric diagnosis, or a general lack of exposure to language (Bishop, 1992; Leonard, 1998). While the estimated prevalence of children with SLI is about 5\% (Law, Boyle, Harris, Harkness, \& Nye, 1998), less than $1 \%$ of Dutch children attend these special schools. When the children entered the special school, they were tested for hearing and intelligence. No significant hearing loss was reported and standardized intelligence tests indicated a level of normal intelligence (standard score was above 85 in all cases).

The control group, matched on gender, consisted of 25 children with typical speech- and language development ( 9 female, 16 male) with a mean age of 5.9 years $(S D=0.5$, range 5 years to 6 years and 5 months), who had no hearing problems. Twenty control children were right-handed, four were left-handed, and one was ambidextrous, based on an adapted version of the questionnaire by Oldfield (1971).

The SLI and control group did not differ in age or non-verbal IQ as measured by the Coloured Progressive Matrices $(t(48)=.14$, $p>.05 ; t(48)=1.54, p>.05$, respectively; Raven, 1965$)$. All participants were monolingual, native speakers of Dutch and had normal or corrected-to-normal vision. They had not yet received any formal reading education and were from middle-income homes. Parents of all children signed the informed consent in accordance with the declaration of Helsinki. The study was approved by the Medical Ethical Committee (CMO Region Arnhem-Nijmegen, The Netherlands).

\subsubsection{Stimuli}

2.1.2.1. Linguistic stimuli. For the linguistic contrast, the naturally produced Dutch words / kan/and/pan/ were used (meaning 'jug' and 'pot' in English, respectively). The words were repeatedly spoken by a native Dutch female speaker and digitally recorded $(44.1 \mathrm{kHz}$, stereo) in a sound-attenuated room. From these recordings, we chose eight exemplars per word, so that our materials contain some typical natural speech variation. Using the Pitch-SynchronousLinear-Overlap-and-Add (PSOLA) algorithm from PRAAT (version 4.5.12), we adapted the duration of the sounds so that the mean duration of the eight utterances of / kan/ (namely, $379 \mathrm{~ms}$ ) was

\footnotetext{
1 A child is diagnosed with SLI if:

I. He/she scores $\leq-1.5$ standard deviations on 2 out of 4 the following aspects (which can not be attributed to a cognitive impairment): 1 . Speech production; 2 . Auditory processing; 3. Grammatical abilities; 4. Lexical-semantic abilities.

II. He/she scores $<-2$ standard deviations on a general test for speech- and language problems (which cannot be attributed to a cognitive impairment), he/she has had speech therapy for at least 6 months without showing any progress, is limited in participating in education, and support and supervision at a mainstream school is not sufficient for the child.
}
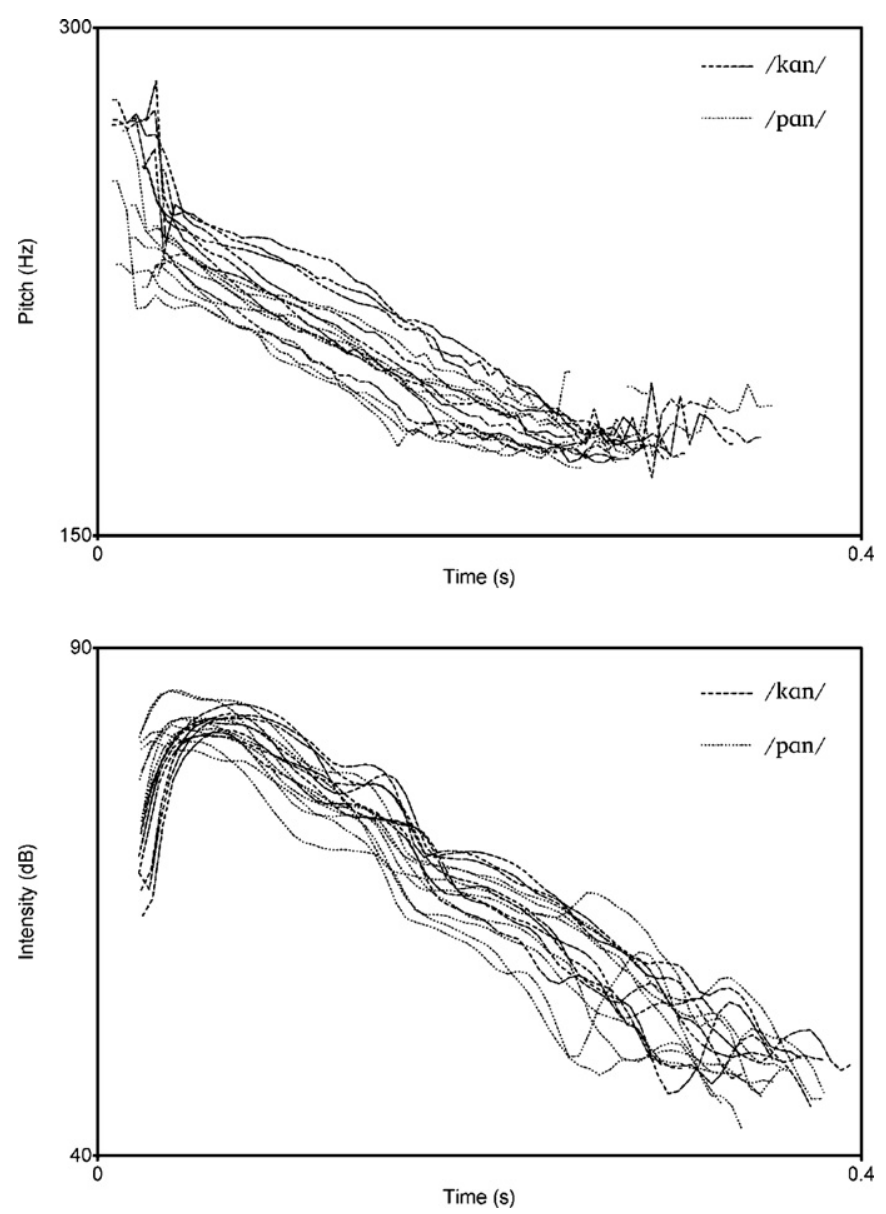

Fig. 1. Pitch and intensity contours of the eight utterances of / kan/ and /pan/ used as linguistic stimuli. The $/ \mathrm{kan} / \mathrm{stimuli}$ are represented by the dashed lines and the /pan/ stimuli by the dotted lines.

comparable to the mean duration of the eight utterances of /pan/ (namely, $377 \mathrm{~ms}$ ). Fig. 1 shows the pitch and intensity contours for each utterance. While there is some variation within the different utterances of a word, there are no gross differences between the words / kan/and/pan/. The linguistic stimuli were filtered with a cutoff at $4000 \mathrm{~Hz}$ (which did not affect the naturalness of the stimuli), in order to be able to create non-linguistic stimuli that were similar in acoustic complexity. The stimuli / kan/and/pan/ mainly differed in the first $50 \mathrm{~ms}$ of the sounds, encompassing the burst spectra and the transitions of the second and third formant into the vowel. Real words were used instead of syllables, such as /ba/, since it is easier for young children to point to the picture corresponding to a word than to a form corresponding to a non-word (Locke, 1993; Seymour, Baran, \& Peaper, 1981). For ecological validity, natural spoken stimuli were used instead of synthesized stimuli. The linguistic stimuli / kan/and/pan/ will be referred to as $\mathrm{L}_{/ \mathrm{kan} /}$ and $\mathrm{L}_{/ \text {pan/, }}$, respectively.

2.1.2.2. Non-linguistic stimuli. Non-linguistic stimuli with identical spectro-temporal variation were created by rotating the spectrum of the average linguistic stimuli (Blesser, 1972; Scott and Wise, 2004). Spectral rotation 'mirrors' the spectrogram of a stimulus along a horizontal line around $2000 \mathrm{~Hz}$. Fig. 2 shows an original and a spectrally rotated version of both $/ \mathrm{kan} / \mathrm{and} / \mathrm{pan} /$. In this rotation, frequencies near $0 \mathrm{~Hz}$ become frequencies near $4000 \mathrm{~Hz}$, and frequencies around $1000 \mathrm{~Hz}$ become frequencies around $3000 \mathrm{~Hz}$, and so on. The rotation is especially salient for the /pan/ example, which has in the original, linguistic version a frequency sweep from very 

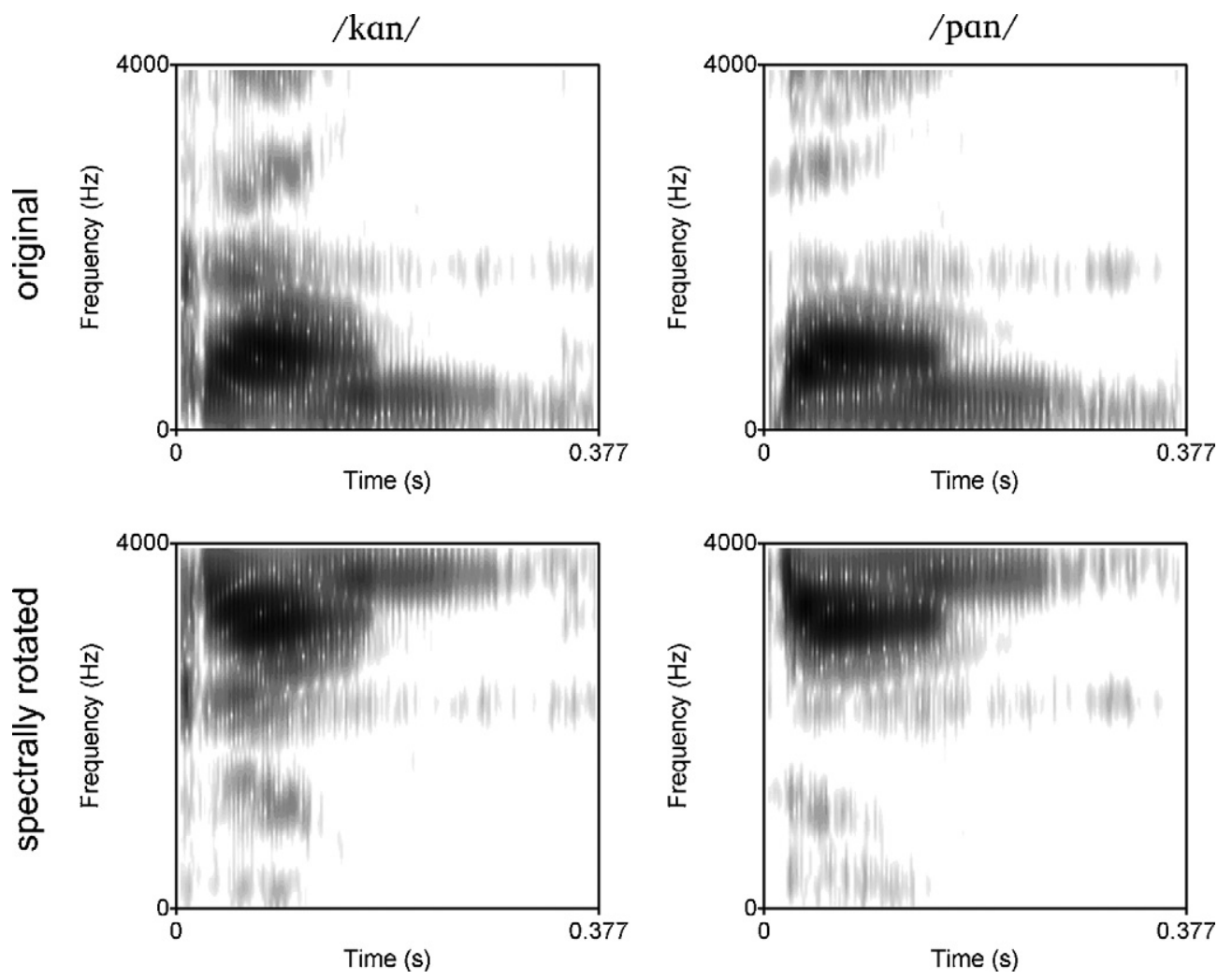

Fig. 2. Original and spectrally rotated version of / kan/ and /pan/ stimuli used as linguistic and non-linguistic stimuli, respectively.

low frequencies to about $1000 \mathrm{~Hz}$ during the initial $50 \mathrm{~ms}$ (see the upper right panel of Fig. 2). In the rotated version, this has become a downwards sweep from frequencies close to the ceiling of $4000 \mathrm{~Hz}$ down to about $3000 \mathrm{~Hz}$. For the non-linguistic stimuli, only one utterance (i.e., the spectrally rotated version of the average utterance of the linguistic stimuli) was used. The non-linguistic stimuli / kan/and/pan/ will be referred to as $\mathrm{NL}_{/ \mathrm{kan} /}$ and $\mathrm{NL}_{\text {/pan/, }}$, respectively.

Before testing children, we pre-tested healthy adults whether the non-linguistic contrast was discriminable at the behavioral level. Ten healthy adults ( 8 female, 2 male; mean age of 26 years), all native speakers of Dutch, performed the Non-Linguistic Discrimination task (described in Section 2.1.3). The mean percentage correct response was $80 \%(S D=15 \%)$. This means that the non-linguistic contrast $\mathrm{NL}_{/ \mathrm{kan} / \text { /pan/ }}$ was clearly discriminable for adults.

\subsubsection{Design and procedure}

Before the children participated in our experimental discrimination tasks, we administered several pre-tests to assess the following language and attention skills of the children: receptive language, productive language, auditory discrimination, rhyme, grapheme knowledge, and attention. The pre-tests were administered to verify that all children with SLI indeed suffered from receptive and productive language problems and to allow us to study how different outcome measures relate to one another. Receptive and productive language was measured by a receptive vocabulary task, and a non-word repetition task respectively. The children were tested individually in a quiet room at school. The tests were administered in three different test sessions. The pre-tests are described in Table 1.

The linguistic and non-linguistic discrimination abilities were tested following Segers and Verhoeven (2005), using a slightly modified version of the Tallal Repetition Task (Tallal \& Piercy, 1973a). Participants had to point to pictures in order to indicate which stimulus or stimuli were presented. For the linguistic stimuli, stimulus $\mathrm{L}_{/ \mathrm{kan} /}$ was represented by a picture of a jug and stimulus $\mathrm{L}_{\text {/pan/ }}$ was represented by a picture of a pot. Since the non-linguistic stimuli had no meaning, pictures of two extraterrestrials were introduced. The stimulus $\mathrm{NL}_{/ \mathrm{kan} /}$ was represented by a picture of an orange extraterrestrial and stimulus $\mathrm{NL}_{\text {/pan/ }}$ by a picture with a different purple extraterrestrial. The participants were told that the extraterrestrials said "hello" in their own extraterrestrial language by means of the $\mathrm{NL}_{/ \mathrm{kan} /}$ or $\mathrm{NL}_{/ \text {pan/ }}$ sound. All stimuli were presented via headphones set at a comfortable hearing level, approximately $60 \mathrm{~dB}$.

The Linguistic and Non-Linguistic Discrimination tasks were both preceded by a familiarization block and an Identification task. In order to keep the children motivated, the Linguistic tasks, that we expected to be easier than the Non-Linguistic tasks, always preceded the Non-Linguistic tasks. The Linguistic and Non-linguistic tasks were administered in two different test sessions. In the Linguistic familiarization block, the participant listened once to each utterance of the linguistic stimuli $\mathrm{L}_{/ \mathrm{kan} /}$ and $\mathrm{L}_{/ \text {pan/ }}$, and in the Non-Linguistic familiarization block, the non-linguistic stimuli $\mathrm{NL}_{\text {/kan/ }}$ and $\mathrm{NL}_{\text {/pan/ }}$ were played eight times. While the participant was listening to the stimuli, the experimenter pointed to the correct picture that belonged to the stimulus. The familiarization block was followed by an Identification task. In the Identification tasks, 16 trials were presented in which one stimulus was played. After listening to each stimulus, the participant was required to point to the picture that belonged to the presented stimulus. The participant received feedback after each trial. This was followed by the Discrimination task, in which 8 practice and 32 test trials of two stimuli with an SOA of $756 \mathrm{~ms}$ were presented. The participant had to point to the pictures corresponding to the stimuli they heard. Trials were scored correct when participants responded to two different pictures for 'different trials' and the same picture twice for 'same trials'. In the Discrimination task, no feedback was given, except for the practice trials. At the start of each trial, participants were prepared for the presentation of 
Table 1

Description of behavioral pre-tests.

\begin{tabular}{|c|c|}
\hline Test & Description \\
\hline Receptive language: vocabulary (Verhoeven \& Vermeer, 1986) & $\begin{array}{l}\text { A word was spoken by the experimenter and the child had to indicate which picture out of } \\
\text { four represented the word. There were } 96 \text { items and after five consecutive incorrect trials the } \\
\text { test was stopped. }\end{array}$ \\
\hline Productive language: pseudoword repetition (Verhoeven, 2006) & $\begin{array}{l}\text { The child had to repeat } 40 \text { pseudowords spoken by the experimenter. The pseudowords } \\
\text { increased in difficulty from /stylt/ (easy) to /bo:marki'tpartant/ (hard). After five incorrect } \\
\text { trials the task was stopped. }\end{array}$ \\
\hline Auditory discrimination (Verhoeven \& Vermeer, 1986) & $\begin{array}{l}\text { The experimenter read aloud two words and the child had to indicate whether the words were } \\
\text { the same or different. The words could differ in a vowel or a consonant which could occur at } \\
\text { all locations: begin, middle, or end of the word. The mouth of the examiner was covered, so } \\
\text { that no cues were available. The test consisted of } 50 \text { items, which were all administered to the } \\
\text { child. }\end{array}$ \\
\hline Rhyme (In den Kleef \& Van Kuyk, 1975) & $\begin{array}{l}\text { The experimenter named a word and the child had to choose out of five pictures which label of } \\
\text { the picture rhymed with the spoken word (or sounded the same, if the concept of rhyme was } \\
\text { unknown). There were } 10 \text { items in this test. }\end{array}$ \\
\hline Grapheme knowledge (In den Kleef \& Van Kuyk, 1975) & $\begin{array}{l}\text { All } 34 \text { Dutch graphemes (including digraphs) were presented to the children on a sheet of } \\
\text { paper. Children had to produce the letter sounds and not the letter names. }\end{array}$ \\
\hline Attention (Manly, Robertson, Anderson, \& Nimmo-Smith, 1999) & $\begin{array}{l}\text { The child was given } 1 \text { min to circle on a crowded road map as many signs of restaurants as } \\
\text { possible. }\end{array}$ \\
\hline
\end{tabular}

the auditory stimuli and their attention was directed toward the pictures.

\subsubsection{Data analysis}

In order to confirm differences in phonological abilities and to compare scores on the Attention, Identification and Discrimination tasks between the SLI and control group, independent samples $t$ tests were conducted. To make sure that the Discrimination task tapped into phonological processing, the relation between scores on this task and on the language pre-tests was studied by calculating correlations over both groups together. In addition, to investigate whether attention skills were related to scores on language tasks, correlations of the Attention task were calculated for both groups together. Finally, to study whether the factors Group (SLI or control group) and Attention Skills both predicted scores on the Discrimination tasks, a stepwise linear regression analysis was performed.

\subsubsection{Results}

After testing 10 children with SLI and 10 children from the control group who all scored at chance level on the Non-Linguistic Identification and Discrimination task, we decided to terminate this part of the experiment. Since the Non-Linguistic tasks turned out to be too difficult for young children, it did not seem to serve any further purpose to test all children on these tasks. The remain- ing 15 children with SLI and 15 control children were tested on all remaining tasks (the pre-tests and the Linguistic Identification and Discrimination tasks). Table 2 shows the average scores of each group of 25 children on the rest of the tests and the results of the independent samples $t$-tests. The SLI group scored significantly below the control group on all language pre-tests. On the Attention task, there was a trend for the SLI group to score below the control group. On both Linguistic Identification and Discrimination tasks, the SLI group performed significantly below the control group.

Correlations between scores on the Linguistic Discrimination task and age and scores on other tests are displayed in the upper part of Table 3. Across groups, the performance on the Linguistic Discrimination task was significantly positively correlated with the performance on all language pre-tests, the Linguistic Identification task, as well as the Attention task. No significant correlation was found with participant age.

Correlations for the Attention task are displayed in the lower part of Table 3. Across groups, there was a significant positive correlation between performance on the Attention task and on all language pre-tests, except for grapheme knowledge. Furthermore, a significant positive correlation was found between Attention scores and scores on both Linguistic Identification and Discrimination tasks. The attention task did not correlate significantly with age.

Table 2

Group scores (mean and SD) for the SLI and control group on pre-tests and Linguistic Identification and Discrimination task.

\begin{tabular}{|c|c|c|c|c|c|}
\hline & Group & Mean & $S D$ & $d$ & Difference between groups $t(48)$ \\
\hline \multirow[t]{2}{*}{ Receptive language } & SLI & 48.04 & 12.34 & -1.68 & $-5.92^{* * *}$ \\
\hline & CG & 67.24 & 10.50 & & \\
\hline \multirow[t]{2}{*}{ Productive language } & SLI & 10.04 & 7.81 & -2.71 & $-9.57^{* * *}$ \\
\hline & CG & 29.20 & 6.27 & & \\
\hline \multirow[t]{2}{*}{ Auditory discrimination } & SLI & 32 & 8.80 & -2.01 & $-3.34^{* *}$ \\
\hline & CG & 39.96 & 8.02 & & \\
\hline \multirow[t]{2}{*}{ Rhyme } & SLI & 6 & 2.52 & -1.62 & $-5.75^{* * *}$ \\
\hline & CG & 9.24 & 1.27 & & \\
\hline \multirow[t]{2}{*}{ Grapheme knowledge } & SLI & 4.80 & 3.50 & -0.83 & $-2.92^{* *}$ \\
\hline & CG & 8.84 & 5.97 & & \\
\hline \multirow[t]{2}{*}{ Attention } & SLI & 8.60 & 4.66 & -0.56 & $-1.96^{p=.055}$ \\
\hline & CG & 11.16 & 4.56 & & \\
\hline \multirow[t]{2}{*}{ Linguistic Identification } & SLI & 15.00 & 1.53 & -0.68 & $-2.39^{*}$ \\
\hline & CG & 15.76 & 0.44 & & \\
\hline \multirow[t]{2}{*}{ Linguistic Discrimination } & SLI & 25.44 & 5.70 & -1.06 & $-3.73^{* *}$ \\
\hline & CG & 29.88 & 1.69 & & \\
\hline
\end{tabular}

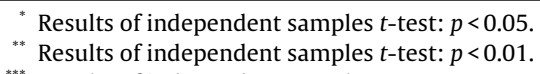

${ }^{* * *}$ Results of independent samples $t$-test: $p<0.001$. 
Table 3

Correlations over both groups for the Linguistic Discrimination task and Attention task.

\begin{tabular}{lc}
\hline Correlation with & Pearson correlation \\
\hline Linguistic discrimination & .26 \\
Age & $.55^{* * *}$ \\
Receptive language & $.57^{* * *}$ \\
Productive language & $.61^{* * *}$ \\
Auditory discrimination & $.55^{* * *}$ \\
Rhyme & $.35^{*}$ \\
Grapheme knowledge & $.43^{* *}$ \\
Attention & $.71^{* * *}$ \\
Linguistic Identification & \\
Attention & .04 \\
Age & $.41^{* *}$ \\
Receptive language & $.39^{* *}$ \\
Productive language & $.34^{*}$ \\
Auditory discrimination & $.35^{*}$ \\
Rhyme & .18 \\
Grapheme knowledge & $.37^{* *}$ \\
Linguistic Identification & $.43^{* *}$ \\
Linguistic Discrimination &
\end{tabular}

$\begin{aligned}{ }^{*} & \text { Results of Pearson correlation: } p<0.05 \\ { }^{* * *} & \text { Results of Pearson correlation: } p<0.01 \\ & \text { Results of Pearson correlation: } p<0.001 \text {. }\end{aligned}$

A stepwise linear regression analysis showed that the two predictors Group (SLI or control group) and Attention Skills together explained a significant portion of variance in scores on the Linguistic Discrimination task $\left(R^{2}=.33 ; F(2,47)=11.31, p<.001\right)$. Participant group significantly predicted Linguistic Discrimination scores when Attention Skills were held constant $(\beta=-.47$, $t(47)=-3.73, p<.01)$. Attention Skills also significantly predicted Linguistic Discrimination scores when Group was held constant $(\beta=.33, t(47)=2.64, p<.05)$.

\subsubsection{Conclusions}

We investigated whether auditory discrimination problems in children with SLI are speech-specific or if they extend to nonspeech information. On the Linguistic Discrimination task, the SLI group performed below the control group. However, the NonLinguistic Discrimination task proved to be too difficult for both controls and children with SLI when tested behaviorally. While these results indicate that children with SLI have a linguistic discrimination problem, we were unable to discern whether these discrimination problems extend to non-speech information. Note, however, that adults tested during a pre-test were able to discriminate the non-linguistic contrast. The difference between the behavioral performance of the adults and children could be due to an immature auditory system of the five-year-olds. However, according to Johnson (2005) there is very little behavioral change in basic sensory abilities between young children and adulthood, which makes it more plausible that the difference between adult and child data are most likely due to meta-cognitive skills, such as attention skills.

Interestingly, data from the Attention task showed that the SLI group tended to have more difficulty in maintaining attention than the control group. Across groups, a positive correlation was found between performance on the Attention task and performance on the Linguistic Discrimination and Identification tasks, as well as on the language pre-tests (except for grapheme knowledge). This means that children who have higher attention skills score better on a variety of language tasks, than children who are poorer in maintaining their attention. A regression analysis showed that both individual's attention skills and their language development predicted the scores of the children on the Linguistic Discrimination task.

To conclude, performances on behavioral tasks are influenced by attention skills. For this reason, and because the behavioral Non-
Linguistic Discrimination task was too difficult for these young children, the nature of auditory discrimination problems will be further studied by means of an MMN paradigm, measuring automatic responses of the brain. This paradigm has two advantages over a behavioral discrimination task. First, the MMN indicates preconscious information processing. This means that if the MMN is elicited for a specific contrast, participants can perceive, at least at a preconscious level, the difference between the stimuli. This is particularly interesting given the difficulty we observed in obtaining reliable measures of non-linguistic discrimination skills in the children. Another advantage of the MMN paradigm is that it does not require participant motivation or attention to the auditory stimuli. Thus, impaired attention will not influence the results. This follow-up study is described in Experiment 2.

\section{Experiment 2}

\subsection{Method}

\subsubsection{Participants}

The same participants were tested as in Experiment 1.

\subsubsection{Stimuli}

The same stimuli were used as in Experiment 1. A pre-test with 16 healthy adults was performed to test whether these stimuli were able to elicit MMNs (see Section 3.2.1).

\subsubsection{Design and procedure}

The participants were tested individually while seated in front of a computer screen. Their EEG was recorded while they were watching a silent movie. The participants were instructed to watch the movie and that they did not have to pay attention to the auditory stimuli. Stimuli were presented via headphones set at a comfortable hearing level, approximately $60 \mathrm{~dB}$. The test session took place in a mobile EEG-lab (a mini-van with a custom-built EEG suite). This made it possible to test children near school. EEG sessions occurred one week after the behavioral test sessions.

The stimuli were presented in a passive oddball paradigm in which the occurrence of a deviant was $12 \%$. Each linguistic and non-linguistic contrast was presented in four blocks. Within each contrast, stimuli were presented as a standard stimulus in two blocks and as deviant stimulus in the other two blocks. For each unique stimulus, 80 deviant and 587 standard stimuli were presented. The SOA was $756 \mathrm{~ms}$ (same as in the behavioral discrimination task from Experiment 1), which resulted in a duration of 16 min per contrast. The deviants were presented in a pseudorandom order, with a minimum of three standards presented between two occurrences of the deviant. Both linguistic and nonlinguistic contrasts were presented to each participant in one test session. Each block started with the presentation of 16 standard stimuli. The order of the blocks was counterbalanced between participants and participants had a short break between the blocks and after each 2 blocks they got a longer break.

\subsubsection{EEG recording}

The EEG was recorded from 24 active electrodes using the Acticap system with $\mathrm{Ag} / \mathrm{AgCl}$ electrodes (Brain Products, Gilching, Germany). Electrodes were placed on standard electrode sites (F7, F3, Fz, F4, F8, FC5, FC1, FCz, FC2, FC6, C3, Cz, C4, CP5, CP1, CP2, CP6, P7, P3, Pz, P4, P8, O1, and O2). All electrodes were referenced to an electrode placed at the left mastoid. In addition, an electrode was placed at the right mastoid for re-referencing during off-line analysis. To monitor vertical eye movements, electrodes were placed beneath and above the left eye. Horizontal eye movements were monitored by electrodes placed on the left and right external canthi. 
Linguistic condition
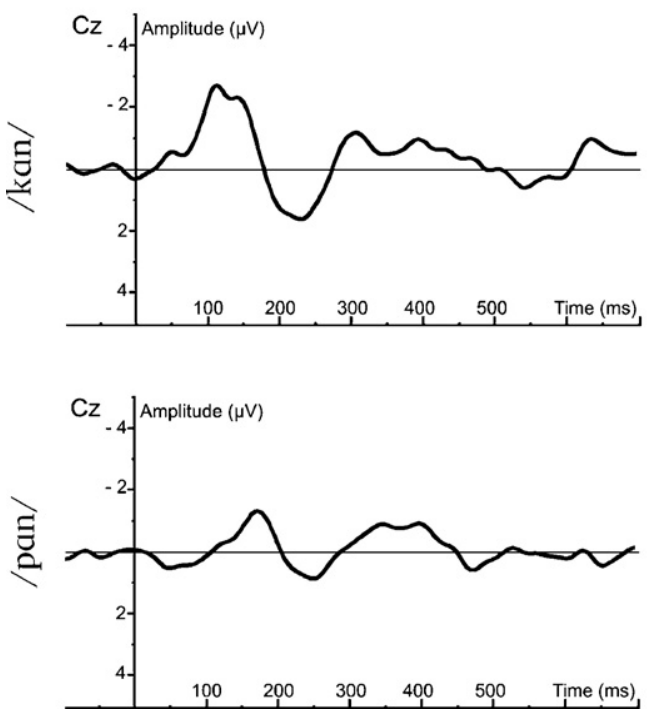

Non-linguistic condition
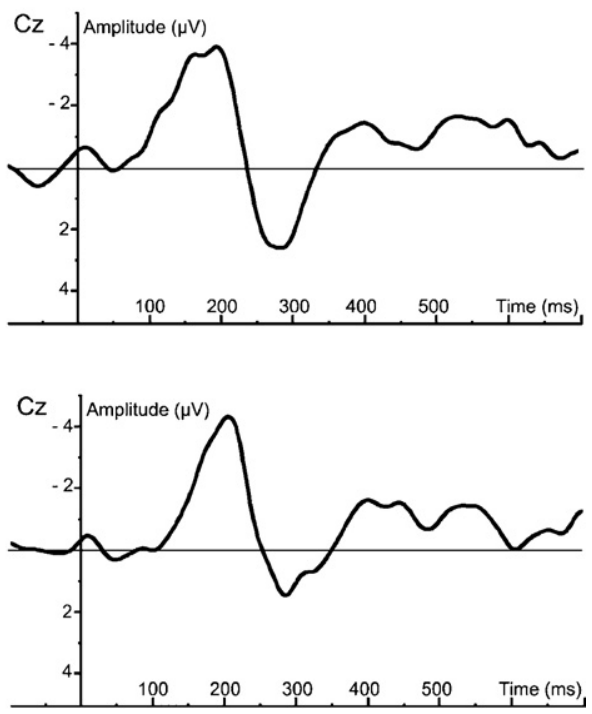

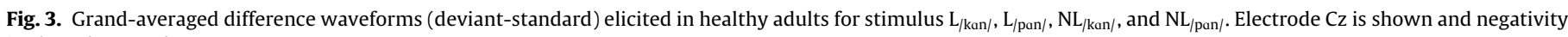
is plotted upwards.

Signals were amplified with BrainAmp DC amplifiers, using a bandpass filter from 1 to $30 \mathrm{~Hz}$ and a sample rate of $500 \mathrm{~Hz}$. Impedances were kept below $20 \mathrm{k} \Omega$ for all electrodes, which is a standard setting for active electrodes. The EEG and EOG were recorded and digitized using Brain Vision Recorder software (1.03, Brain Products, Gilching, Germany).

\subsubsection{Data analysis}

EEG data were re-referenced to linked mastoids and an automatic ocular correction was performed (Gratton, Coles, \& Donchin, 1983). The signal was segmented in time windows ranging from $100 \mathrm{~ms}$ before stimulus onset to $700 \mathrm{~ms}$ after stimulus onset, and baseline corrected relative to a 50 -ms pre-stimulus interval. Segments with artifacts exceeding $\pm 100 \mu \mathrm{V}$ in any channel were excluded from further analysis. Remaining segments were averaged for each condition and for each participant. For each stimulus, difference waveforms were calculated using the difference between two ERPs elicited by the same stimulus (e.g., $\mathrm{L}_{/ \mathrm{kan} /}$ as a deviant stimulus $-\mathrm{L}_{/ \mathrm{kan} /}$ as a standard stimulus). The ERP of a given stimulus serving as a standard immediately preceding a deviant was hence subtracted from the ERP of the same stimulus serving as a deviant. The EEG signals were analyzed using Brain Vision Analyzer software (1.05.0002, Brain Products, Gilching, Germany).

To test whether the MMNs of the SLI group differed from those of the control group on the linguistic and/or non-linguistic contrast, a GLM repeated-measures analysis of variance (ANOVA) was applied to the mean amplitude of the ERPs during a specific time window based on visual inspection at electrode $\mathrm{Cz}$. The within-subjects factors were Contrast (two levels: linguistic and non-linguistic) and Stimulus type (two levels: standard and deviant) and the between-subjects factor was Group (two levels: SLI and controls). Greenhouse-Geisser correction for violation of sphericity assumption was applied when appropriate (Greenhouse \& Geisser, 1959).

\subsection{Results}

\subsubsection{Pre-test with adults}

Before ERPs were recorded from the children, we pre-tested whether both linguistic and non-linguistic contrasts were able to elicit an MMN in healthy adults. Sixteen healthy adults (10 female, 6 male; mean age of 23 years) were tested on a passive oddball paradigm. ${ }^{2}$ Fig. 3 shows the difference waveforms belonging to each stimulus at electrode $\mathrm{Cz}$, where the differences were maximal. The ANOVA revealed a significant effect of Stimulus type for $\mathrm{L}_{/ \mathrm{kan} /}, \mathrm{NL}_{/ \mathrm{kan} /}$, and $\mathrm{NL}_{/ \mathrm{pan} /}\left(F(1,15)=27.24, p<.001, \eta_{p}^{2}=.65\right.$; $F(1,15)=22.08, p<.001, \eta_{p}^{2}=.60 ; F(1,15)=62.81, p<.001, \eta_{p}^{2}=.81$, respectively), but no significant effect of Stimulus type was shown for $\mathrm{L}_{/ \mathrm{pan} /}\left(F(1,15)=3.61, p>.05, \eta_{p}^{2}=.19\right)$. This pre-test shows that the / kan/ deviant is able to elicit large and significant MMNs in both linguistic and non-linguistic conditions in adults, whereas the /pan/ deviant only elicits a large and significant MMN in the nonlinguistic condition. ${ }^{3}$ Therefore, we only analyzed the ERPs elicited by the / kan/ deviant in the study with children.

\subsubsection{SLI group vs. control group}

For the SLI group, on average, $69(S D=11)$ and $71(S D=8)$ artifact-free trials remained for the linguistic standard and deviant stimulus, respectively. For the non-linguistic standard and deviant stimulus, the number of remaining trials was, on average, 69 $(S D=11)$ and $70(S D=10)$, respectively. For the control group, in the linguistic condition, a mean of $69(S D=12)$ and $70(S D=10)$ trials remained for the standard and deviant stimulus, respectively. For the non-linguistic condition, a mean of $70(S D=9)$ and $69(S D=12)$ artifact-free trials remained for the standard and deviant stimulus, respectively.

\footnotetext{
2 A highly comparable design, procedure, EEG recording, and EEG preprocessing was used as described in Section 3.1. The only differences were: (a) for the adults the nose was used as online reference and the EEG signal was re-referenced to the average of the left and the right mastoid electrodes; (b) segments with artifacts exceeding $\pm 75 \mu \mathrm{V}$ in any channel were rejected for further analysis. For the data analysis, difference waveforms were calculated for each stimulus $\mathrm{L}_{/ \mathrm{kan} /}, \mathrm{L}_{/ \text {pan/ }}, \mathrm{NL}_{/ \mathrm{kan} /}$, and $\mathrm{NL}_{\text {/pan/ }}$ by subtracting the ERP of the standard stimulus from the ERP of the same stimulus presented as a deviant stimulus. A repeated-measures ANOVA was applied to the mean amplitude of the difference wave at electrode $\mathrm{Cz}$ in a time window of $50 \mathrm{~ms}$ centered around the grand-averaged peak of the MMN. The ANOVA was performed for each stimulus separately and included one within-subjects factor Stimulus type (two levels: standard and deviant).

3 Such asymmetric MMNs have been reported previously for coronal vs. noncoronal stimuli, which has been attributed to a phonological underspecification of coronal stimuli (Eulitz \& Lahiri, 2004). The current stimuli are both non-coronal (/k/ is velar, /p/ is labial). These results show that MMN asymmetries may be more common than previously assumed.
} 
Linguistic condition
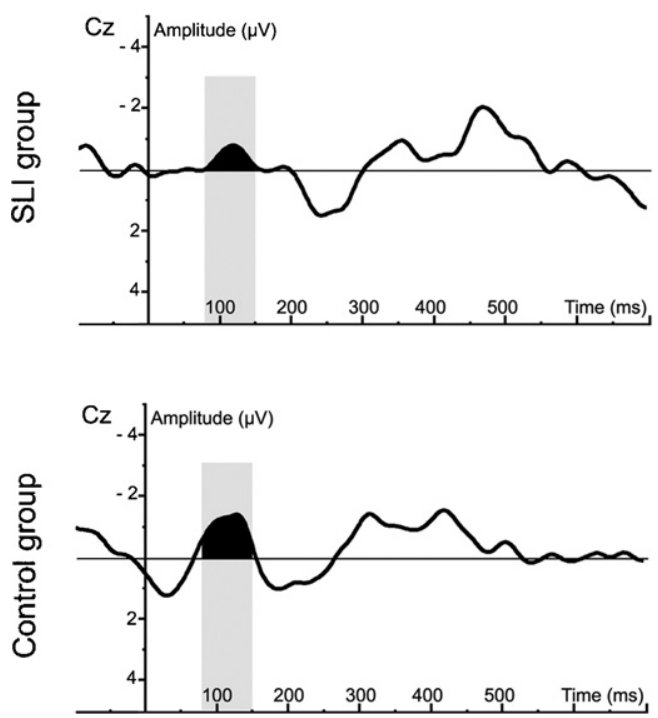

Non-linguistic condition
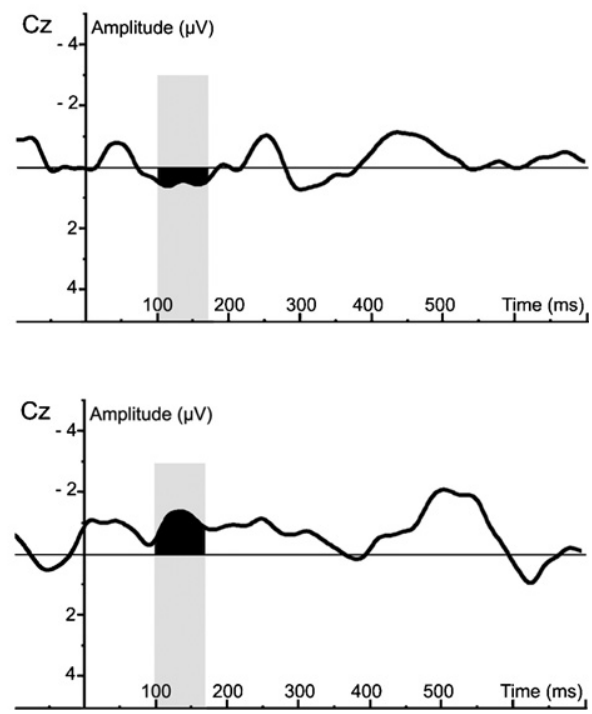

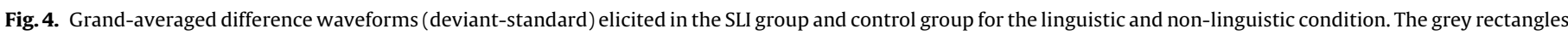
show the time window to which a repeated-measures ANOVA is applied. Electrode $\mathrm{Cz}$ is shown and negativity is plotted upwards.

Fig. 4 presents the grand-averaged difference waveforms at electrode $\mathrm{Cz}$, where the differences were maximal. This figure shows that, in the linguistic condition, a negative going difference is elicited in the control group between 80 and $150 \mathrm{~ms}$, while this difference is smaller in the SLI group. For the non-linguistic condition, a negative going difference is elicited in the control group between 100 and $170 \mathrm{~ms}$. This difference seems to be absent in the SLI group. The time windows described above were used for statistical analyses, i.e., $80-150 \mathrm{~ms}$ for the linguistic contrast and $100-170 \mathrm{~ms}$ for the non-linguistic contrast.

The repeated-measures ANOVA revealed that there was a significant main effect of Stimulus type $\left(F(1,48)=4.35, p<.05, \eta_{p}^{2}=\right.$ .08 , corresponding to an effect of $-0.6 \mu \mathrm{V}, S D=3.3)$, and a significant interaction between Stimulus type and Group $(F(1,48)=4.26$, $\left.p<.05, \eta_{p}^{2}=.08\right)$. There was no significant interaction between Stimulus type and Contrast $\left(F(1,48)=.49, p>.05, \eta_{p}^{2}=.01\right)$ or between Stimulus type, Contrast, and Group $(F(1,48)=.51, p>.05$, $\left.\eta_{p}^{2}=.01\right)$. This indicates that the difference between the ERPs of the standard and deviant stimulus differed between the groups. A repeated-measures ANOVA performed for the groups separately showed that, for the control group, there was a significant main effect of Stimulus type $\left(F(1,24)=8.43, p<.01, \eta_{p}^{2}=.26\right.$, corresponding to an effect of $-1.2 \mu \mathrm{V}, S D=3.1)$ and no significant interaction between Stimulus type and Contrast $\left(F(1,24)=.00, p>.05, \eta_{p}^{2}<\right.$ .01). In contrast, for the SLI group there was no significant main effect of Stimulus type $\left(F(1,24)=.00, p>.05, \eta_{p}^{2}<.01\right)$, nor a significant interaction between Stimulus type and Contrast $(F(1,48)=.89$, $\left.p>.05, \eta_{p}^{2}=.04\right)$.

\subsection{Conclusions}

In Experiment 2, we used an MMN paradigm in which automatic responses of the brain were measured in order to study the nature of auditory discrimination problems in children with SLI. In the control group, both linguistic and non-linguistic contrasts elicited significant MMNs. In the SLI group, however, neither the linguistic nor the non-linguistic contrasts elicited a significant MMN. This means that children with SLI are impaired in linguistic as well as non-linguistic information processing as compared to the control group.

\section{General discussion}

The present study investigated the nature of auditory discrimination problems in children with SLI. More specifically, we were interested in whether these problems are primarily phonological in nature, or whether they coincide with non-speech processing difficulties. Five-year-old Dutch children diagnosed with SLI having both receptive and productive language problems, were compared to control children with typical speech- and language development matched on age, gender, and non-verbal IQ. To be able to directly compare phonemic and auditory discrimination abilities, we used a linguistic and non-linguistic contrast that had identical acoustically complex changes. Because children with SLI have a high risk of developing attention problems (Beitchman et al., 1990), discrimination abilities were tested in an active behavioral task as well as a passive MMN paradigm.

In Experiment 1, the nature of discrimination problems was investigated by means of an active behavioral discrimination task. In addition, individual attention skills and a variety of language abilities were measured. The results showed that the SLI group performed below the control group on all language tests of receptive language, productive language, auditory discrimination, rhyme, and grapheme knowledge. This confirms that the group of children with SLI in this study were significantly impaired at the level of phonological abilities (cf. Bishop \& Leonard, 2000; Van Weerdenburg, Verhoeven, \& Van Balkom, 2006; Van Weerdenburg, Verhoeven, Van Balkom, \& Bosman, 2009). Moreover, the SLI group had more difficulties with the Linguistic Discrimination task than the control group. Correlation analyses showed that higher scores on standardized tests of receptive language, productive language, general auditory discrimination, rhyme, and/or a grapheme knowledge, predicted higher scores on our Linguistic Discrimination task. No significant correlation with age was found. These correlations signify that our Linguistic Discrimination task taps into phonological ability and that there was no relation between the performance of the children on the Linguistic Discrimination task and their age. In contrast to adults, who showed in a pre-test that they were able to discriminate the non-linguistic contrast at the behavioral level, children from both groups were unable to perform the Non-Linguistic Discrimination task. This suggests that when non-linguistic stimuli are used that are acous- 
tically equally complex as their linguistic counterparts but contain no phonemes, discrimination in an active non-linguistic behavioral task becomes too hard for young children. Since there is very little behavioral change in basic auditory sensory abilities between five-year-old children and adults (Johnson, 2005), the difference between the data of the adults and children is most likely to be explained by meta-cognitive skills, such as attention skills.

Interestingly, in the Attention task, the SLI group seemed to have more difficulty with maintaining attention than the control group. Correlation analyses for the Attention task showed that children who are better in maintaining their attention obtain better scores on the Linguistic Discrimination task and on a large variety of behavioral language tasks, than children who are impaired on attention. The diagnosis of SLI as well as one's attention skills had a predictive value on linguistic discrimination scores. Since it was found that attention skills play a significant role when discrimination abilities are tested behaviorally and that no useful information could be obtained about the non-linguistic discriminarion abilities of the children, we used a passive MMN paradigm to further investigate the nature of discrimination problems.

In Experiment 2, the nature of discrimination problems was studied by means of measuring the automatic responses of the brain using an auditory oddball paradigm. In this paradigm, participants did not have to pay attention to the auditory stimuli. If the MMN component is elicited, this means that participants are able to discriminate stimuli at a preconscious and pre-attentive level. The ERP results showed that, in the control group, an MMN was elicited in both linguistic and non-linguistic contrasts. The MMNs of the linguistic and non-linguistic contrasts did not differ from each other. In the SLI group, however, no MMN was elicited in either contrast. This means that the SLI group was impaired at the neural level in discriminating both speech and non-speech contrasts as compared to the control group. Thus, the phonological deficit seems to coincide with non-speech processing difficulties in children with SLI having receptive as well as productive language problems. This supports accounts that relate the symptoms in children with SLI to a more general problem in non-speech processing difficulties, which has already been suggested by a series of studies (e.g., Alonso-Búa et al., 2006; Farmer \& Klein, 1995; Lachmann et al., 2005; McAnally et al., 2000; Stein \& Walsh, 1997; Tallal \& Piercy, 1973a, 1973b, $1974,1975)$, and is in contrast to studies that found evidence for a primarily speech specific deficit (Meng et al., 2005; Mody et al., 1997; Schulte-Körne et al., 1998; Schulte-Körne et al., 1999; Sharma et al., 2006; Snowling, 2000; Studdert-Kennedy, 2002; StuddertKennedy \& Mody, 1995; Uwer et al., 2002).

This finding may also have practical implications. If discrimination problems in children with SLI are due to general non-speech processing difficulties, one may assume that in order to improve phonological skills, it would be beneficial to train children with a phonological deficit on discriminating linguistic as well as nonlinguistic stimuli (Merzenich et al., 1996). However, to date, transference of training in non-phonological discrimination onto improvement in phonological processing remains an empirical issue.

It is interesting to note that whereas neither controls nor children with SLI were able to discriminate the non-linguistic contrast at the behavioral level, an MMN was elicited in the control group. This suggests that although control children were unable to indicate reliably whether non-linguistic stimuli differed, at the neural level they were able to perceive the difference. However, in the SLI group, no negative going difference was observed in the nonlinguistic contrast. This provides neural evidence for an inability of SLI individuals to distinguish the non-linguistic stimuli at the age of five years old.
Note that the present study has some limitations. First, in our behavioral and neural discrimination tasks, we only used one contrast $/ \mathrm{kan} / \mathrm{vs}$./pan/. In order to study the extent of discrimination impairment in children with SLI it is necessary to include several contrasts in which phonological differences are varied. Second, whereas the present study provides evidence for speech and nonspeech processing difficulties in children with SLI, it does not prove that the linguistic discrimination problems originate from the non-speech processing difficulties. Future longitudinal studies are needed to uncover cause and effect relations between auditory perception, on the one hand, and phonological variables, on the other. Third, the role of attention skills in behavioral discrimination tasks could have been studied more thoroughly, by including an auditory attention task (but care must be taken to avoid confounds with auditory processing difficulties) and questionnaires for teachers and parents (Conners, Siteranios, Parker, \& Epstein, 1998a; Conners, Siteranios, Parker, \& Epstein, 1998b). This would be recommendable for future research. In addition, if time permitted, a working memory task could be included to make the behavioral test battery more complete.

In conclusion, optimal assessment of auditory discrimination problems requires the use of contrasts that contain equally complex acoustic changes. At the behavioral level, it turns out be highly complicated to assess to what extent children perceive differences in these complex sound contrasts. However, a passive MMN paradigm, in which the brain's automatic responses are recorded to measure discrimination skills at a preconscious level, provides more insight into the underlying problem. This is especially the case since elicitation of the MMN component does not require attention, which according to the behavioral results of this study can influence scores on discrimination tasks. Using the MMN paradigm, the present study provides further support for an account that relates the symptoms in children with SLI to non-speech processing difficulties, coinciding with a phonological deficit.

\section{Acknowledgments}

We would like to thank Irene Janssen and Marieke de Peuter for helping with collecting the data. Barbara van Wagensveld and Petra van Alphen are acknowledged for thinking along with us setting up the study and for voicing the stimuli. Thanks to Gary Turner for helpful comments. The study is funded by the European Union, Interreg 4-BMG-V-1 = 31 and Viataal (St. Michielsgestel, The Netherlands).

\section{References}

Alonso-Búa, B., Díaz, F., \& Ferraces, M. J. (2006). The contribution of AERPs (MMN and LDN) to studying temporal vs. linguistic processing deficits in children with reading difficulties. International Journal of Psychophysiology, 59, 159-167.

Beitchman, J. H., Hood, J., \& Inglis, A. (1990). Psychiatric risk in children with speech and language disorders. Journal of Abnormal Child Psychology, 18, 283-296.

Bird, J., Bishop, D. V. M., \& Freeman, N. H. (1995). Phonological awareness and literacy development in children with expressive phonological impairments. Journal of Speech and Hearing Research, 38, 446-462.

Bishop, D. V. M. (1992). The underlying nature of specific language impairment. Journal of Child Psychology and Psychiatry and Allied Disciplines, 33, 3-66.

Bishop, D. V. M. (1997). Uncommon understanding: Development and disorders of language comprehension in children. Hove, England: Psychology Press.

Bishop, D. V. M. (2007). Using mismatch negativity to study central auditory processing in developmental language and literacy impairments: Where are we, and where should we be going? Psychological Bulletin, 133, 651-672.

Bishop, D. V. M., \& Adams, C. (1990). A prospective study of the relationship between specific language impairment, phonological disorders and reading retardation. Journal of Child Psychology and Psychiatry, 31, 1027-1050.

Bishop, D. V. M., \& Leonard, L. B. (2000). Speech and language impairments in children: Causes, characteristics, intervention and outcome. Hove: Psychology Press.

Bishop, D. V. M., \& Snowling, M. J. (2004). Developmental dyslexia and specific language impairment: Same or different? Psychological Bulletin, 130, $858-886$. 
Blesser, B. (1972). Speech perception under conditions of spectral transformation: I. Phonetic characteristics. Journal of Speech and Hearing Research, 15, 5-41.

Conners, C. K., Siteranios, G., Parker, J. D. A., \& Epstein, J. N. (1998a). The revised Conners' Parent Rating Scale (CPRS-R): Factor structure, reliability, and criterion validity. Journal of Abnormal Child Psychology, 26, 257-268.

Conners, C. K., Siteranios, G., Parker, J. D. A., \& Epstein, J. N. (1998b). Revision and restandardization of the Conners' Teaching Rating Scale (CTRS-R): Factor structure, reliability, and criterion validity. Journal of Abnormal Child Psychology, 26, 279-291.

Eulitz, C., \& Lahiri, A. (2004). Neurobiological evidence for abstract phonological representations in the mental lexicon during speech recognition. Journal of Cognitive Neuroscience, 16, 577-583.

Farmer, M. E., \& Klein, R. M. (1995). The evidence for a temporal processing deficit linked to dyslexia: A review. Psychonomic Bulletin and Review, 2, 460-493.

Gratton, G., Coles, M. G., \& Donchin, E. (1983). A new method for off-line removal of ocular artifact. Electroencephalography Clinical Neurophysiology, 55, 468-484.

Greenhouse, S. W., \& Geisser, S. (1959). On methods in the analysis of profile data. Psychometrika, 24, 95-112.

In den Kleef, T., \& Van Kuyk, J. (1975). Curriculum Schoolrijpheid deel 2B. Den Bosch: Malmberg.

Johnson, M. H.(2005). Developmental cognitive neuroscience: An introduction. Malden, MA: Wiley-Blackwell.

Lachmann, T., Berti, S., Kujala, T., \& Schröger, E. (2005). Diagnostic subgroups of developmental dyslexia have different deficits in neural processing of tones and phonemes. International Journal of Psychophysiology, 56, 105-120.

Law, J., Boyle, J., Harris, F., Harkness, A., \& Nye, C. (1998). Screening for primary speech and language delay: A systematic review of the literature. International Journal of Language E' Communication Disorders, 33, 21-23.

Leonard, L. B. (1998). Children with specific language impairment. Cambridge, MA: MIT Press.

Locke, J. L. (1993). The child's path to spoken language. Cambridge, MA: Harvard University Press.

Manly, T., Robertson, I. H., Anderson, V., \& Nimmo-Smith, I. (1999). The Test of Everyday Attention for Children (TEA-Ch). Bury St. Edmunds: Thames Valley Test Company.

McAnally, K. I., Castles, A., \& Stuart, G. W. (2000). Visual and auditory processing impairments in subtypes of developmental dyslexia: A discussion. Journal of Developmental and Physical Disabilities, 12, 145-156.

Meng, X., Sai, X., Wang, C., Wang, J., Sha, S., \& Zhou, X. (2005). Auditory and speech processing and reading development in Chinese school children: Behavioural and ERP evidence. Dyslexia: An International Journal of Research and Practice, 11, 292-310.

Merzenich, M. M., Jenkins, W. M., Johnston, P., Schreiner, C., Miller, S. L., \& Tallal, P. (1996). Temporal processing deficits of language-learning impaired children ameliorated by training. Science, 271, 77-81.

Mody, M., Studdert-Kennedy, M., \& Brady, S. (1997). Speech perception deficits in poor readers: Auditory processing or phonological coding? Journal of Experimental Child Psychology, 64, 199-231.

Näätänen, R., Gaillard, A. W., \& Mantysalo, S. (1978). Early selective-attention effect on evoked potential reinterpreted. Acta Psychologica, 42, 313-329.

Näätänen, R., Paavilainen, P., Rinne, T., \& Alho, K. (2007). The mismatch negativity (MMN) in basic research of central auditory processing: a review. Clinical Neurophysiology, 118, 2544-2590.

Oldfield, R. C. (1971). The assessment and analysis of handedness: The Edinburgh inventory. Neuropsychologia, 9, 97-113.

Ramus, F. (2001). Dyslexia. Talk of two theories. Nature, 412, 393-395.

Ramus, F., Rosen, S., Dakin, S. C., Day, B. L., Castellote, J. M., White, S., et al. (2003). Theories of developmental dyslexia: Insights from a multiple case study of dyslexic adults. Brain, 126, 841-865.
Raven, J. C. (1965). Guide to using the coloured progressive matrices. London: H.K. Lewis \& Co.

Robinson, R. J. (1991). Causes and associations of severe and persistent specific speech and language disorders in children. Developmental Medicine E Child Neurology, 33, 943-962.

Rosen, S. (2003). Auditory processing in dyslexia and specific language impairment: Is there a deficit? What is its nature? Does it explain anything? Journal of Phonetics, 31, 509-527.

Schulte-Körne, G., Deimel, W., Bartling, J., \& Remschmidt, H. (1998). Auditory processing and dyslexia: Evidence for a specific speech processing deficit. Neuroreport: An International Journal for the Rapid Communication of Research in Neuroscience, 9, 337-340.

Schulte-Körne, G., Deimel, W., Bartling, J., \& Remschmidt, H. (1999). The role of phonological awareness, speech perception, and auditory temporal processing for dyslexia. European Child E Adolescent Psychiatry, 8, III/28-III/34.

Scott, S. K., \& Wise, R. J. S. (2004). The functional neuroanatomy of prelexical processing in speech perception. Cognition, 92, 13-45.

Segers, E., \& Verhoeven, L. (2005). Effects of lengthening the speech signal on auditory word discrimination in kindergartners with SLI. Journal of Communication Disorders, 38, 499-514.

Serniclaes, W., Sprenger-Charolles, L., Carre, R., \& Demonet, J.-F. (2001). Perceptual discrimination of speech sounds in developmental dyslexia. Journal of Speech, Language, and Hearing Research, 44, 384-399.

Seymour, C. M., Baran, J. A., \& Peaper, R. E. (1981). Auditory discrimination: Evaluation and intervention. In N. J. Lass (Ed.), Speech and language: Advances in basic research and practice (pp. 1-58). New York/London: Academic Press.

Sharma, M., Purdy, S. C., Newall, P., Wheldall, K., Beaman, R., \& Dillon, H. (2006) Electrophysiological and behavioral evidence of auditory processing deficits in children with reading disorder. Clinical Neurophysiology, 117, 1130-1144.

Snowling, M. J. (2000). Dyslexia. Malden, MA: Blackwell Publishing.

Stein, J., \& Walsh, V. (1997). To see but not to read; the magnocellular theory of dyslexia. Trends in Neurosciences, 20, 147-152.

Studdert-Kennedy, M. (2002). Deficits in phoneme awareness do not arise from failures in rapid auditory processing. Reading and Writing, 15, 5-14.

Studdert-Kennedy, M., \& Mody, M. (1995). Auditory temporal perception deficits in the reading-impaired: A critical review of the evidence. Psychonomic Bulletin $\mathcal{E}$ Review, 2, 508-514

Tallal, P., \& Piercy, M. (1973a). Defects of non-verbal auditory perception in children with developmental aphasia. Nature, 241, 468-469.

Tallal, P., \& Piercy, M. (1973b). Developmental aphasia: impaired rate of non-verba processing as a function of sensory modality. Neuropsychologia, 11, 389-398.

Tallal, P., \& Piercy, M. (1974). Developmental aphasia: Rate of auditory processing and selective impairment of consonant perception. Neuropsychologia, 12, 83-93.

Tallal, P., \& Piercy, M. (1975). Developmental aphasia: The perception of brief vowels and extended stop consonants. Neuropsychologia, 13, 69-74.

Uwer, R., Albrecht, R., \& von-Suchodoletz, W. (2002). Automatic processing of tones and speech stimuli in children with specific language impairment. Developmental Medicine and Child Neurology, 44, 527-532.

Van Weerdenburg, M. W. C., Verhoeven, L., \& Van Balkom, H. (2006). Towards a typology of specific language impairment. Journal of Child Psychology and Psychiatry, 47, 176-189.

Van Weerdenburg, M. W. C., Verhoeven, L., Van Balkom, H., \& Bosman, A. (2009). Cognitive and linguistic precursors to early literacy achievement in children with specific language impairment. Scientific Studies of Reading, 13, 484-507.

Verhoeven, L. (2006). Screeningstest voor Taal- E Leesproblemen. Arnhem: Cito.

Verhoeven, L., \& Vermeer, A. (1986). Taaltoets Allochtone Kinderen. Handleiding Tilburg: Zwijsen. 